\title{
Avaliação da consulta de enfermagem aos pacientes com tuberculose na atenção
} primária à saúde

\author{
Déborah Raquel Carvalho de Oliveira ${ }^{1}$, Bertha Cruz Enders ${ }^{2}$, \\ Caroline Evelin Nascimento Kluczynick Vieira ${ }^{3}$, Larissa Soares Mariz ${ }^{4}$
}

\author{
${ }^{1}$ Enfermeira, Mestre em Enfermagem. \\ Discente do Programa de Pós-Graduação \\ em Enfermagem, nível Doutorado, da \\ Universidade Federal do Rio Grande do \\ Norte (UFRN). Natal, RN, Brasil. E-mail: \\ deborahrco@hotmail.com. \\ ${ }^{2}$ Enfermeira, Doutora em Enfermagem \\ Professora Colaboradora do Programa de \\ Pós-Graduação em Enfermagem da UFRN. \\ Natal, RN, Brasil. E-mail: bertha@ufrnet.br. \\ ${ }^{3}$ Enfermeira, Mestre em Enfermagem. \\ Discente do Programa de Pós-Graduação \\ em Enfermagem, nível Doutorado, da \\ UFRN. Natal, RN, Brasil. E-mail: \\ carolinekluczynik@gmail.com. \\ ${ }^{4}$ Enfermeira, Mestre em Enfermagem. \\ Discente do Programa de Pós-Graduação \\ em Enfermagem, nível Doutorado, da \\ UFRN. Natal, RN, Brasil. E-mail: \\ larissamariz@gmail.com.
}

Recebido: 30/10/2014.

Aceito: 12/08/2015.

Publicado: 31/03/2016.

Como citar esse artigo:

Oliveira DRC, Enders BC, Vieira CENK, Mariz LS. Avaliação da consulta de enfermagem aos pacientes com tuberculose na atenção primária à saúde. Rev. Eletr. Enf. [Internet]. 2016 [acesso em: _____ ] 18:e1153.

Disponível em:

http://dx.doi.org/10.5216/ree.v18.32593.

\section{RESUMO}

Este estudo objetivou avaliar a consulta de enfermagem aos pacientes com tuberculose. Estudo descritivo, quantitativo, realizado em município do nordeste brasileiro, com 60 enfermeiros da Atenção Primária à Saúde, mediante aplicação de questionário baseado no protocolo da consulta de enfermagem ao paciente com tuberculose do Ministério da Saúde. Elementos da coleta de dados eram mais abordados, ocorrendo, na anamnese e exame físico, a supervalorização das queixas (100\% sempre investigavam) e da mensuração do peso do paciente (96,8\% realizavam). Os diagnósticos de enfermagem, planejamento, implementação e avaliação do plano terapêutico não eram realizados completamente e aspectos socioculturais eram subestimados. Essa fragmentação pode contribuir como deficiência na operacionalização do Plano de Controle da Tuberculose. A Consulta de Enfermagem, se ampliada científica e tecnologicamente pode favorecer o desenvolvimento dos programas de saúde e a minimização de entraves que permeiam o controle da Tuberculose.

Descritores: Cuidados de Enfermagem; Atenção Primária à Saúde; Tuberculose.

\section{INTRODUÇÃO}

A Consulta de Enfermagem (CE) é uma importante ferramenta de trabalho do enfermeiro, que utiliza componentes do método científico para uma assistência sistematizada de enfermagem ${ }^{(1-3)}$. 
A CE deve ser desenvolvida em todos os locais de saúde em que ocorra a assistência do enfermeiro ${ }^{(1)}$. O enfermeiro deve desenvolvê-la de forma sistematizada e um dos meios de Sistematização da Assistência de Enfermagem (SAE) é o Processo de Enfermagem (PE) ${ }^{(4)}$.

Estudo desenvolvido na Inglaterra ${ }^{(5)}$ descreve que a CE sistematizada, com avaliação do plano terapêutico, investigação da vida do paciente e orientações sobre a condução do tratamento, além da valorização de fatores socioculturais do paciente, passa a ser um instrumento capaz de assegurar acesso aos serviços de saúde e continuidade de determinado tratamento.

Dentro da Atenção Primária à Saúde (APS), um dos programas a serem conduzidos pelo enfermeiro é o Programa de Controle da Tuberculose (TB). Nesse processo, o abandono do tratamento pelo paciente tem sido considerado um dos principais entraves nesse controle e pode estar relacionado a condições socioeconômicas, como a baixa escolaridade, alcoolismo, uso de drogas, além de relações fragmentadas com o serviço de saúde que o atende ${ }^{(6)}$.

De acordo com o Protocolo de Enfermagem no Tratamento Diretamente Observado (TDO) da Tuberculose na Atenção Básica do Ministério da Saúde (MS), a Consulta de Enfermagem configura-se como um instrumento do cuidado e deve seguir as etapas do PE: identificação de problemas de saúde do cliente ou coleta de dados (com anamnese e exame físico), delineamento dos Diagnósticos de Enfermagem (DEs), planejamento e implementação das ações e a avaliação do plano ${ }^{(7)}$.

Apesar de as diretrizes postuladas pelo MS e as normatizações do COFEN acerca da CE serem conhecidas, estudos que abordam o cuidar do enfermeiro ao paciente com TB registram que o cuidado realizado envolve ações de busca ativa e tratamento medicamentoso, mas que estas não estão centradas em um cuidado integral ${ }^{(8-10)}$.

Há de se pensar no processo que o enfermeiro utiliza ao realizar a sua consulta aos pacientes com TB. Acredita-se que essa consulta, quando sistematizada e focada no paciente ${ }^{(5)}$, não apenas oferece uma oportunidade de estabelecer vínculo, mas permite conhecer melhor o indivíduo e suas dificuldades para o planejamento e desenvolvimento de ações de acompanhamento.

A CE deve estar fundamentada cientificamente em uma visão de clínica ampliada, e envolver a agregação de conhecimentos científicos e competências próprias. No Reino Unido, por exemplo, tem-se observado que os pacientes apresentam receio nas consultas com o enfermeiro, pondo em questão o seu saber científico e atuação na condução de tratamentos ${ }^{(11)}$.

Portanto, é preciso que os profissionais avancem na perspectiva de utilizar o PE para sistematizar a assistência de enfermagem. É relevante compreender que o PE, envolve etapas específicas. Caso contrário, a Enfermagem estará embasando o seu cuidado em um saber limitado e fragmentado.

Apesar do crescimento das discussões e publicações sobre a SAE e PE, principalmente a partir da década de 70, observa-se na literatura, que os registros de pesquisa abordam, em sua maioria, a implantação e experiência da utilização do PE nos níveis secundários e terciários de atenção à saúde no Brasil(12), sendo poucas as publicações nos serviços de atenção primária. 
A exemplo disto, cita-se a realidade de Portugal, também descrita ainda como falha, uma vez que na realidade dos serviços de saúde deste país pouco se observa os registros de enfermagem feitos em conformidade com o $\mathrm{PE}^{(13)}$.

Reconhece-se, então, a importância que o enfermeiro se aproprie da instrumentalização do PE na Atenção Primária à Saúde, fortalecendo o vínculo entre profissional/usuário bem como uma assistência acolhedora e humanizada ${ }^{(14)}$.

Questiona-se: Quais elementos do Processo de Enfermagem são desenvolvidos pelo enfermeiro ao realizar a consulta de enfermagem ao paciente com TB?

Assim sendo, o objetivo deste estudo foi avaliar os elementos da Consulta de Enfermagem aos pacientes com TB na Atenção Primária à Saúde.

A identificação desses elementos na prática do enfermeiro poderá elucidar até que ponto esses profissionais se valem de princípios científicos inerentes às competências do enfermeiro para o desenvolvimento do PE na Atenção Primária.

\section{MÉTODO}

Estudo descritivo, com abordagem quantitativa. É parte de um estudo mais amplo intitulado: "A prática do enfermeiro da atenção primária à saúde nas consultas ao paciente com tuberculose". Foi desenvolvido nas unidades de Atenção Primária à Saúde de um município do Nordeste brasileiro.

A população foi composta por 156 enfermeiros atuantes nas unidades de APS. Uma amostra de 60 enfermeiros foi definida por meio de cálculo amostral para populações finitas ${ }^{(15)}$ e estratificada proporcionalmente entre os cinco distritos sanitários do Município: Norte I, Norte II, Leste, Oeste e Sul. Desse modo, a representação de enfermeiros por distrito foi assim definida: Norte I - 13; Norte II - 14; Leste - 7; Oeste-15; e Sul-11.

O critério de seleção dos enfermeiros era de que tivessem desenvolvido ou estivessem desenvolvendo, no período de coleta, a consulta ao paciente com TB. Foram excluídos enfermeiros que referiram nunca ter realizado consulta a paciente com TB.

A seleção foi acidental em cada distrito sanitário. As unidades de saúde pertencentes a cada distrito eram visitadas para identificação dos enfermeiros que atendessem o critério de seleção até atingir o número determinado de sujeitos para aquele distrito.

Para a coleta de dados, utilizou-se um questionário estruturado, desenvolvido a partir das diretrizes contidas no Protocolo de Enfermagem para o Tratamento Diretamente Observado da Tuberculose na Atenção Básica, do Ministério da Saúde ${ }^{(7)}$.

O questionário continha, além das questões de caracterização profissional, 35 questões referentes à frequência com que o enfermeiro realizava ações relacionadas ao PE durante a consulta ao paciente com TB. Foram investigadas variáveis referentes às seguintes ações do enfermeiro: anamnese do paciente; desenvolvimento do exame físico do paciente; diagnósticos de enfermagem; planejamento de ações; 
implementação do plano terapêutico e na avaliação dos resultados obtidos. As respostas eram fornecidas segundo as opções 1 "Sempre", 2 "Às vezes" e 3 "Nunca.

$O$ questionário foi submetido a pré-teste com três enfermeiras da $A B$ para avaliação aparente do conteúdo e para constatar a compreensão gerada pela sua aplicação. Após o pré-teste houve pequenas alterações nos termos utilizados no instrumento, de forma a evitar interpretações equivocadas nos questionamentos propostos. Os questionários utilizados no pré-teste não fizeram parte da amostra do estudo.

A coleta de dados foi realizada no período de setembro a outubro de 2014. Uma vez identificados como possíveis sujeitos, os enfermeiros eram contatados previamente para averiguação de sua disponibilidade e do horário adequado para responder ao questionário. Os questionários eram preenchidos e devolvidos logo em seguida ao pesquisador.

A coleta foi desenvolvida pelo pesquisador principal e acadêmicos de enfermagem dos 4 , 6 으 e 8 은 períodos do curso. Ressalta-se que todos foram treinados quanto à abordagem dos participantes, aplicação do questionário e importância da leitura e assinatura do TCLE.

O estudo foi autorizado pelo Comitê de Ética em Pesquisa da Universidade Federal do Rio Grande do Norte, em consonância com a legislação vigente ${ }^{(16)}$, com registro $n=31266314.9 .0000 .5537$.

Os dados foram analisados por meio de estatística descritiva e do Programa IBM SPSS 20.0. e apresentados em frequência relativa.

\section{RESULTADOS}

Quanto à caracterização profissional dos sujeitos da pesquisa, $66,1 \%$ trabalhavam em USF, 22,6\% pertenciam a UBS e $8,1 \%$ trabalhavam em unidade mista de saúde.

As ações desenvolvidas nas consultas de enfermagem ao paciente com TB foram avaliadas de acordo com o PE e seus elementos: investigação/coleta de dados; levantamento dos DEs; planejamento das ações; implementação; e avaliação. Dentro desses elementos foram abordados itens considerados pelo Ministério da Saúde para composição da consulta de enfermagem ao paciente com TB.

Quanto aos elementos da coleta de dados, a Tabela 1 demonstra os elementos abordados e a frequência de utilização destes por parte dos enfermeiros na consulta ao paciente com TB. 
Tabela 1: Elementos de investigação dos problemas de saúde do paciente abordados pelos enfermeiros da APS na consulta ao paciente com tuberculose $(n=60)$. Natal, RN, Brasil, 2014.

\begin{tabular}{|c|c|c|c|}
\hline \multirow{2}{*}{ Variáveis } & \multicolumn{3}{|c|}{ Frequência } \\
\hline & Sempre \% & Às vezes \% & Nunca \% \\
\hline Realização da anamnese & 100 & - & - \\
\hline Levantamento de queixas & 100 & - & - \\
\hline Levantamento sobre sintomas da doença & 93,3 & 6,7 & - \\
\hline Levantamento de aspectos clínicos, epidemiológicos e psicossociais & 36,7 & 51,7 & 11,7 \\
\hline Levantamento sobre padrão alimentar & 98,3 & 1,7 & - \\
\hline Levantamento sobre história anterior de TB & 80,0 & 20,0 & - \\
\hline Levantamento sobre histórico familiar de TB & 81,7 & 18,3 & - \\
\hline Levantamento sobre condições de moradia & 26,7 & 73,3 & - \\
\hline Levantamento sobre Uso de álcool/drogas ilícitas ou outras substâncias & 95,0 & 5,0 & - \\
\hline Levantamento sobre Histórico de abandono do tratamento & 71,7 & 28,3 & - \\
\hline Levantamento sobre Sofrimento relacionado ao estigma/preconceito da doença & 20,0 & 36,7 & 43,3 \\
\hline Levantamento sobre Alterações na rotina de trabalho em decorrência da doença & 18,3 & 46,7 & 35,0 \\
\hline Solicitação de exames & 63,3 & 36,7 & - \\
\hline Avaliação de exames anteriores para identificação do estado atual da doença & 40,0 & 38,3 & 21,7 \\
\hline Realização do exame físico do paciente & 65,0 & 35,0 & - \\
\hline Inspeção geral da pele & 68,3 & 31,7 & - \\
\hline Avaliação do turgor cutâneo & 40,0 & 38,3 & 21,7 \\
\hline Mensuração do peso & 100 & - & - \\
\hline Inspeção das mucosas & 46,7 & 43,3 & 10,0 \\
\hline Avaliação da frequência respiratória & 45,0 & 40,0 & 15,0 \\
\hline Ausculta torácica e cardíaca & 33,3 & 40,0 & 26,7 \\
\hline Percussão torácica & 16,7 & 38,3 & 45,0 \\
\hline Registro da anamnese e exame físico no prontuário & 68,3 & 31,7 & - \\
\hline
\end{tabular}

Sobre o levantamento dos diagnósticos de enfermagem durante a CE ao paciente com TB, foram observados os seguintes resultados, conforme a Figura 1:

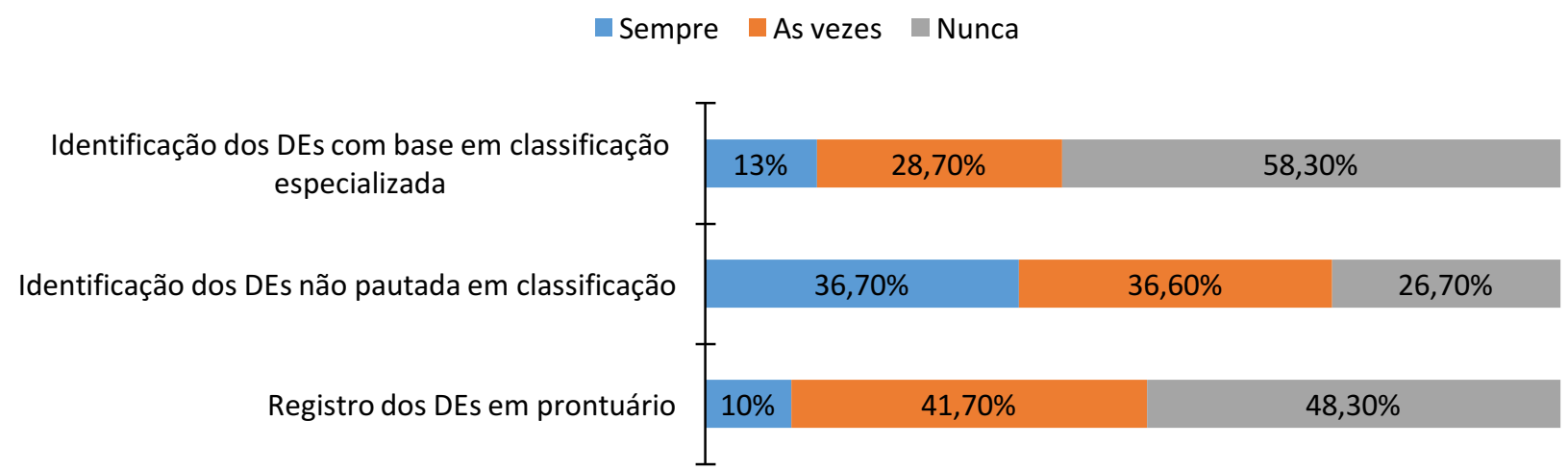

Figura 1: Elementos do levantamento de diagnósticos de enfermagem realizados pelos enfermeiros da APS na consulta ao paciente com tuberculose ( $n=60)$. Natal, RN, Brasil, 2014.

Os elementos do planejamento das ações, implementação e avaliação do plano de cuidados estão apresentados na Figura 2. 


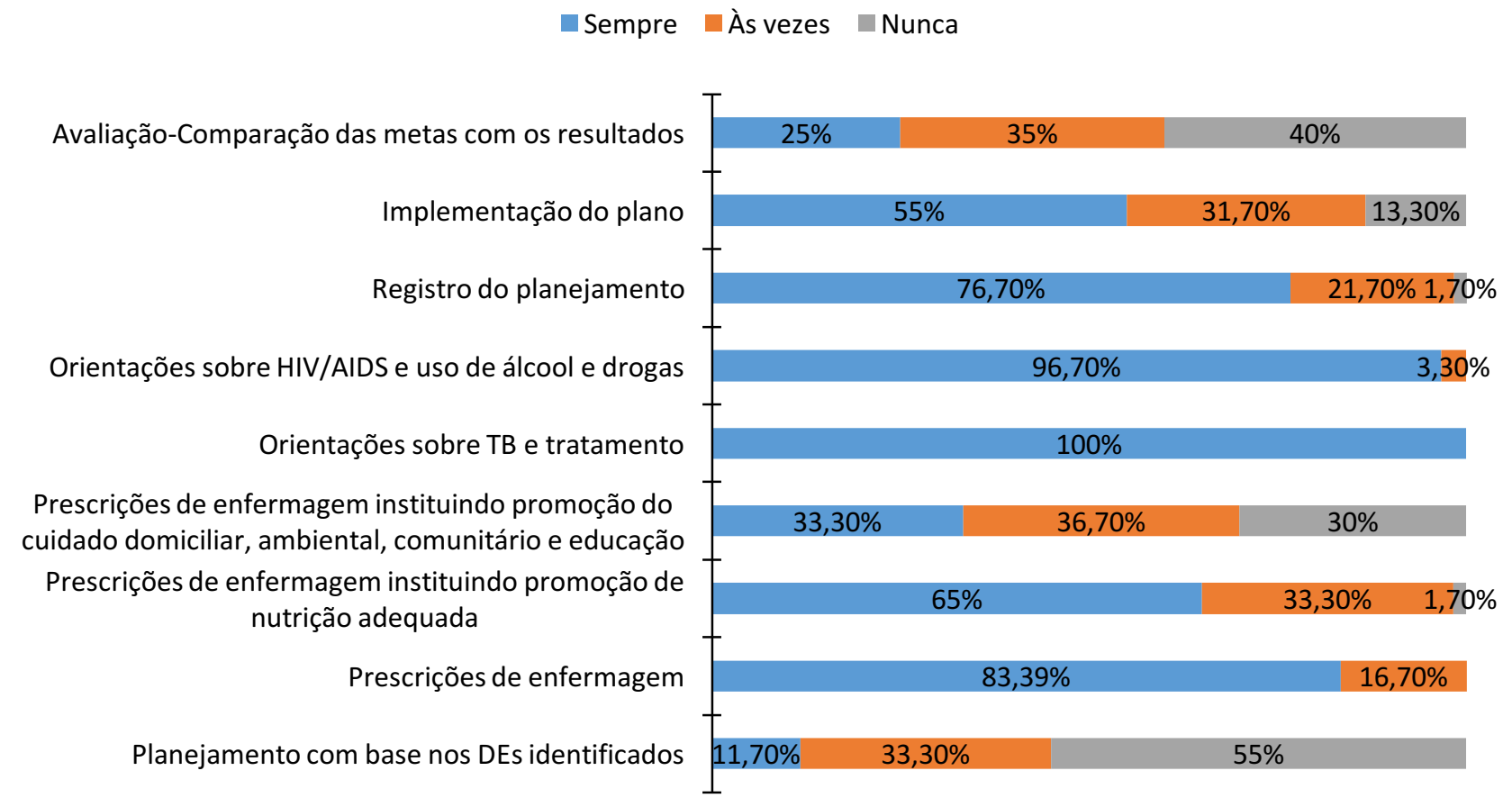

Figura 2: Elementos relacionados ao planejamento das ações, implementação e avaliação do plano de cuidados realizados pelos enfermeiros na CE ao paciente com TB ( $n=60)$. Natal, RN, Brasil, 2014.

\section{DISCUSSÃO}

No que diz respeito à atenção ao paciente com tuberculose, o Ministério da Saúde ${ }^{(7)}$ recomenda que o PE seja realizado nas consultas de enfermagem ao paciente com TB, o que, neste estudo, apresentou uma fragmentação, tendo em vista que apenas alguns elementos desse método eram executados na prática do enfermeiro da APS.

Sobre os elementos da coleta de dados (Tabela 1), os enfermeiros afirmaram que realizavam a anamnese (ou entrevista) do paciente, porém, esta anamnese estava voltada para o levantamento de queixas físicas dos pacientes. Outros aspectos como a investigação de sofrimento do paciente relacionado a estigma sobre a doença e outros aspectos socioculturais eram menos abordados.

A Consulta de Enfermagem, todavia, deve ser um instrumento capaz de abarcar não apenas necessidades específicas, mas deve ser um espaço para o enfrentamento de outras demandas do cotidiano, como é o caso de estigmas e entraves culturais que permeiam algumas doenças, a exemplo da TB, hanseníase, que dentro do Programa de Controle na APS, demanda em fragmentação no cuidar ${ }^{(17)}$.

A supervalorização dos aspectos físicos e biológicos decorre das raízes de um modelo tradicional que permeia a atenção à saúde humana, onde a prioridade é dada a partir de aspectos biológicos e físicos do paciente ${ }^{(18)}$.

Destaca-se, ainda, que, para que haja posteriormente uma identificação dos problemas do paciente para a formulação dos DEs e seja feita a tomada de decisões, deve-se haver o reconhecimento de todo o conjunto de dados necessários e existentes em torno do paciente, ou seja, todas as evidências existentes devem ser identificadas a partir da coleta de dados sobre o problema de saúde do paciente ${ }^{(19)}$. 
Outros programas preconizados no âmbito da APS, como saúde da mulher e da criança também apontam que o enfermeiro apresenta dificuldades em suas consultas, reconhecendo a necessidade de maior preparo e que educação em saúde é entendida como informação em saúde ${ }^{(3)}$.

Quanto à realização do exame físico, foi observado que alguns métodos eram deixados de lado pelos enfermeiros, sobretudo a ausculta e percussão torácica. Isso pode estar relacionado a algumas dificuldades em promover uma clínica ampliada por parte da enfermagem. Algumas ações, como o exame físico mais detalhado são atrelados ao exame médico, o que culmina em uma reprodução do modelo hegemônico ${ }^{(20)}$.

O paciente com TB, especialmente com a forma pulmonar, deve ter uma atenção concentrada ao seu sistema respiratório inferior. A ausculta torácica constitui uma importante ferramenta para avaliação do estado de saúde do paciente, inclusive na identificação de possíveis agravamentos.

Os elementos relacionados à identificação de DEs, planejamento das ações, implementação e avaliação, apresentaram-se com maior fragmentação no desenvolvimento do PE durante as consultas de enfermagem.

Os DEs, por exemplo, foram apontados por uma minoria como sendo identificados de acordo com a classificação especializada de Enfermagem. Outra parte dos sujeitos afirmou identificá-los, porém sem base em uma taxonomia própria.

Os diagnósticos de enfermagem devem ser identificados de modo a iniciar o processo de julgamento clínico do paciente com TB e, de acordo com o Ministério da Saúde, devem ser identificados com base na taxonomia NANDA, devido a sua reconhecida utilidade clínica na prática de enfermagem ${ }^{(7)}$.

É importante ressaltar, ainda, que, apesar de a taxonomia da NANDA surgir como a preconizada pelo MS e a classificação que mais aparece na literatura para a identificação de $D E s^{(21)}$, a Classificação Internacional das Práticas de Enfermagem em Saúde Coletiva (CIPESC) ${ }^{(22)}$ representa uma importante ferramenta no âmbito da APS.

Nesse sentido, destaca-se a necessidade de identificar as práticas do enfermeiro na APS e fazer o aprofundamento dessa prática com as ferramentas metodológicas de trabalho da enfermagem, como as classificações de enfermagem, a fim de possibilitar uma linguagem padronizada para o cuidado de enfermagem ${ }^{(20)}$.

Além da não identificação dos diagnósticos de enfermagem, o registro dos DEs não era feito nos prontuários dos pacientes, o que constitui outra quebra na sistematização da consulta, pois fere as diretrizes postuladas pelo Conselho Federal de Enfermagem, que determina que os registros de todas as etapas sejam feitos nos prontuários.

No estudo apresentado, conforme os Gráficos 01 e 02, os enfermeiros, em sua maioria, assim como não levantavam DEs para os pacientes com TB, não realizavam sempre o planejamento de suas ações com base em DEs. Realizavam prescrições de enfermagem direcionadas à doença do paciente, porém não investiam nas prescrições que visassem à comunidade em que este paciente estava inserido, tampouco à educação em saúde. 
Nesse sentido, torna-se necessário lembrar que a educação em saúde bem como a instituição da promoção do cuidado domiciliar podem ser fatores de acesso e continuidade de determinado tratamento, por aumentarem o vínculo com o profissional ${ }^{(5)}$.

A implementação do plano terapêutico junto, bem como a avaliação dos resultados, também se apresentaram de forma fragmentada, o que compromete a atenção a este paciente, pois inibem uma reavaliação das condutas direcionadas a ele, e, tendo em vista a inserção em contextos que permitem uma inconstância no seu tratamento, pode culminar no abandono do tratamento ${ }^{(10)}$.

No sentido da não utilização do PE na prática do enfermeiro, destaca-se que alguns fatores contribuem para tal, dentre eles o déficit no conhecimento acerca do PE, e grandes demandas nos serviços ${ }^{(23)}$.

Destaca-se também a necessidade do compromisso das gestões e supervisões com a atualização dos profissionais para que o PE seja incorporado à prática de enfermagem. A educação permanente é uma estratégia facilitadora para o desenvolvimento do PE pelos enfermeiros, pois amplia o conhecimento teórico sobre seus elementos, principalmente pois, como observado no estudo, a maioria dos enfermeiros tem um longo período desde que se graduaram, e isso é um fator que reflete no despreparo teórico e prático para o desenvolvimento do $\mathrm{PE}^{(23)}$.

\section{CONCLUSÃO}

No estudo foi verificado uma fragmentação do processo de enfermagem na Consulta de Enfermagem ao paciente com tuberculose, onde alguns elementos do PE eram enfatizados durante as consultas, como aspectos da anamnese e exame físico, em detrimento do levantamento de diagnósticos de enfermagem, planejamento, implementação e avaliação.

No que diz respeito aos diagnósticos de enfermagem, foram apontados como tendo sido levantados em alguns momentos sem o uso de classificação especializada. Todavia, não se sabe como ocorreu esse diagnóstico, uma vez que não foi explorada a maneira como ocorria e se estava fundamentado cientificamente. Isso pode configurar uma limitação do estudo. Além disso, o fato de os enfermeiros emitirem as respostas aos questionamentos pode não traduzir a realidade de alguns pontos registrados no prontuário.

Os resultados encontrados permitem a reflexão de uma prática fragmentada nas consultas de enfermagem, o que pode contribuir como deficiência na operacionalização do Plano de Controle da Tuberculose. A Consulta de Enfermagem, se ampliada científica e tecnologicamente pode favorecer o desenvolvimento dos programas de saúde e a minimização de entraves que permeiam o controle da Tuberculose.

Outros estudos devem ser realizados a fim de verificar a Consulta de Enfermagem junto a outras populações, haja vista o processo de enfermagem ser assegurado como direito e dever na prática de enfermagem. 


\section{REFERÊNCIAS}

1. Resolução COFEN 358/2009 (BR) [Interrnet]. Dispõe sobre a Sistematização da Assistência de Enfermagem e a implementação do Processo de Enfermagem em ambientes, públicos ou privados, em que ocorre o cuidado profissional de Enfermagem, e dá outras providências. COFEN. 15 out 2009 [acesso em: 31 Mar. 2016]. Disponível em: http://www.cofen.gov.br/resoluo-cofen-3582009_4384.html.

2. Oliveira SKP, Queiroz APO, Matos DPM, Moura AF, Lima FET. Temas abordados na consulta de enfermagem: revisão integrativa da literatura. Rev Bras Enferm [Internet]. 2012 [acesso em: 31 Mar. 2016];65(1):155-61. Disponível em: http://dx.doi.org/10.1590/S0034-71672012000100023.

3. Campos RMC, Ribeiro CA, Silva CV, Saparolli ECL. Consulta de enfermagem em puericultura: a vivência do enfermeiro na Estratégia de Saúde da Família. Rev Esc Enferm USP [Internet]. 2011 [acesso em: 31 Mar. 2016];45(3):566-74. Disponível em: http://dx.doi.org/10.1590/50080-62342011000300003.

4. Barros ALBL, Lopes JL. A legislação e a sistematização da assistência de enfermagem. Enfermagem em Foco [Internet]. 2010 [acesso em: 31 Mar. 2016];1(2):63-5. Disponível em:

http://revista.portalcofen.gov.br/index.php/enfermagem/article/view/17.

5. Stenner KL, Courtenay M, Carey N. Consultations between nurse prescribers and patients with diabetes in primary care: A qualitative study of patient views. Int J Nurs Stud [Internet]. 2011 [acesso em: 31 Mar. 2016];48(1):37-46. Disponível em: http://dx.doi.org/10.1016/j.ijnurstu.2010.06.006.

6. Chirinos NEC, Meirelles BHS. Fatores associados ao abandono do tratamento da tuberculose: uma revisão integrativa. Texto Context - Enferm [Internet]. 2011 [acesso em: 31 Mar. 2016];20(3):399-406. Disponível em: http://dx.doi.org/10.1590/\$0104-07072011000300023.

7. Ministério da Saúde. Tratamento Diretamente Observado (TDO) da Tuberculose na Atenção Básica: Protocolo de Enfermagem [Internet]. Brasília: Ministério da Saúde; 2011 [acesso em: 31 Mar. 2016]. Disponível em:

http://bvsms.saude.gov.br/bvs/publicacoes/tratamento diretamente observado tuberculose.pdf.

8. Sá LD, Oliveira AAV, Gomes ALC, Nogueira JA, Villa TCS, Collet N. aring for tuberculosis patients in the Family Health Strategy: the nurses' perceptions. Rev Esc Enferm USP [Internet]. 2012 [acesso em: 31 Mar. 2016];46(2):356-63. Disponível em: http://dx.doi.org/10.1590/S0080-62342012000200013.

9. Barrêto AJR, Evangelista ALF, Sá LD, Almeida SA, Nogueira JA, Lopes AMC. Gestão do cuidado à tuberculose: da formação à prática do enfermeiro. Rev Bras Enferm [Internet]. 2013 [acesso em: 31 Mar. 2016];66(6):847-53. Disponível em: http://dx.doi.org/10.1590/\$0034-71672013000600006.

10. Alves RS, Souza KMJ, Oliveira AAV, Palha PF, Nogueira JA, Sá LD. Tuberculosis treatment abandonment and comprehensive health care to patients in the family healthcare strategy. Texto Context - Enferm [Internet]. 2012 [acesso em: 31 Mar. 2016];21(3):650-7. Disponível em: http://dx.doi.org/10.1590/s0104-07072012000300021. 11. Rashid C. Benefit sand limitations of nurses taking on aspects of the clinical role of doctors in primary care: integrative literature review. J Adv Nurs [Internet]. 2010 [acesso em: 31 Mar. 2016];66(8):1658-70. Disponível em: http://dx.doi.org/10.1111/j.1365-2648.2010.05327.x.

12. Cavalcante RB, Otoni A, Bernardes MFVG, Cunha SGS, Santos C da S, Silva PC da. Experiências de sistematização da assistência de enfermagem no Brasil: um estudo bibliográfico. Revista de Enfermagem da UFSM [Internet]. 2011 [acesso em: 31 Mar. 2016];1(3):461-71. Disponível em: http://dx.doi.org/10.5902/217976922832.

13. Silva MA, Graveto J. Modelo Conceptual Versus "Modelo Oculto" para a (na) Prática da Enfermagem. Pensar Enfermagem [Internet]. 2008 [acesso em: 31 Mar. 2016];12(2):67-70. Disponível em:

http://pensarenfermagem.esel.pt/files/2008_12_2_67-70.pdf.

14. Varela GC, Fernandes SCA. Conhecimentos e práticas sobre a sistematização da assistência de enfermagem na Estratégia Saúde da Família. Cogitare Enferm [Internet]. 2013 [acesso em: 31 Mar. 2016];18(1):124-30. Disponível em: http://dx.doi.org/10.5380/ce.v18i1.31317.

15. Barbetta PA, Reis MM, Bornia AC. Estatística para Cursos de Engenharia e Informática. 3å ed. São Paulo: Atlas; 2010.

16. Resolução № 466 do Conselho Nacional de Saúde, de 12 de dezembro de 2012 (BR). Aprova as diretrizes e normas regulamentadoras de pesquisas envolvendo seres humanos. Diário Oficial da União. 12 dez 2012 [acesso em: 31 Mar. 2016]. Disponível em: http://bvsms.saude.gov.br/bvs/saudelegis/cns/2013/res0466_12_12_2012.html.

17. Duarte MTC, Ayres JA, Simonetti JP. Consulta de enfermagem: estratégia de cuidado ao portador de hanseníase em atenção primária. Texto Context - Enferm [Internet]. 2009 [acesso em: 31 Mar. 2016];18(1):100-7. Disponível em: 
http://dx.doi.org/10.1590/S0104-07072009000100012.

18. Souza KMJ, Sá LD, Palha PF, Nogueira JA, Villa TCS, Figueiredo DA. Abandono do tratamento de tuberculose e relações de vínculo com a equipe de saúde da família. Rev Esc Enferm USP [Internet]. 2010 [acesso em: 31 Mar. 2016];44(4):904-10. Disponível em: http://dx.doi.org/10.1590/50080-62342010000400007.

19. Bittencourt GKGD, Crossetti MGO. Critical thinking skills in the nursing diagnosis process. Rev Esc Enferm USP [Internet]. 2013 [acesso em: 31 Mar. 2016];47(2):341-7. Disponível em: http://dx.doi.org/10.1590/5008062342013000200010 .

20. Matumoto S, Fortuna CM, Kawata LS, Mishima SM, Pereira MJB. Nurses' clinical practice in primary care: a process under construction. Rev Lat Am Enfermagem [Internet]. 2011 [acesso em: 31 Mar. 2016];19(1):123-30. Disponível em: http://dx.doi.org/10.1590/\$0104-11692011000100017.

21. North American Nursing Diagnosis Association. Diagnósticos de enfermagem da NANDA: definições e classificação 2009-2011. 1a engl. ed. Garcez RM, translator. Porto Alegre: Artmed; 2010.

22. Cubas MR, Egry EY. Classificação Internacional de Práticas de Enfermagem em Saúde Coletiva - CIPESC ${ }^{\circledR}$. Rev Esc Enferm USP [Internet]. 2008 [acesso em: 31 Mar. 2016];42(1):181-6. Disponível em: http://dx.doi.org/10.1590/S008062342008000100024.

23. Menezes SRT, Priel MR, Pereira LL. Autonomia e vulnerabilidade do enfermeiro na prática da Sistematização da Assistência de Enfermagem. Rev Esc Enferm USP [Internet]. 2011 [acesso em: 31 Mar. 2016];45(4):953-8. Disponível em: http://dx.doi.org/10.1590/s0080-62342011000400023. 